\title{
AVALIAÇÃO DOS COEFICIENTES DE SEGURANÇA DOS CÓDIGOS NORMATIVOS PARA REFORÇOS AO CISALHAMENTO EM VIGAS DE CONCRETO ARMADO REFORÇADAS COM PRFC
}

\author{
Assessments of safety factors of current models for CFRP shear \\ strengthened reinforced concrete beams
}

\author{
Lunna Alexia Uno ${ }^{1}$, Adriano Vieira Risson ${ }^{2}$, Nara Villanova Menon ${ }^{3}$
}

Recebido em 21 de novembro de 2015; recebido para revisão em 28 de janeiro de 2016; aceito em 24 de março de 2016; disponível on-line em 11 de maio de 2016.

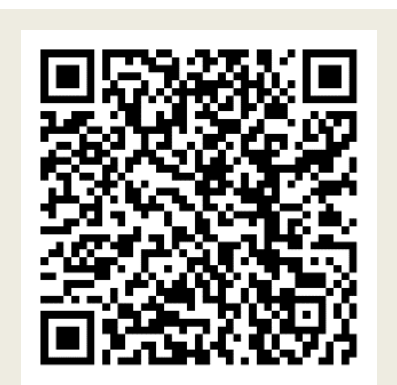

PALAVRAS CHAVE:

Vigas de concreto armado;

Reforço ao cisalhamento; PRF - (Polímeros Reforçados com Fibras); Modelos de análise; Coeficientes de segurança.

\section{KEYWORDS:}

$R C$ beams;

Shear strengthening; FRP (fibre-reinforced polymers); Analytical models; Safety factors.

\footnotetext{
* Contato com os autores:

${ }^{1}$ e-mail: lunna.alexia@gmail.com (L. A. Uno)

Discente do curso de Engenharia Civil da Universidade Estadual de Maringá.

2e-mail: adrianorisson@hotmail.com (A. V. Risson)

Engenheiro Civil, Mestrando PCV Estruturas, Universidade Estadual de Maringá.

3 e-mail: nvmenon@uem.br ( N. V. Menon)

Engenheira Civil, Doutora, Professora Associada, Universidade Estadual de Maringá.

ISSN: 2179-0612 D.O.I. 10.5216/reec.V11i3.35586
}

\begin{abstract}
RESUMO: O problema da avaliação do cisalhamento em vigas de concreto armado quantificam o cisalhamento por meio de uma simples soma das contribuiç concreto (Vc), do aço (VsW), e do PRF (Vf). Apresentar comparaçôs ent obtidos de resultados provenientes de investigações experimentais é o objetivo (2008), fib BULLETIN 14 - TG 9.3 (2001) e CNR-DT 200 (2004). A base de dado feita pelos resultados experimentais de 30 vigas de concreto armado reforçadas. que algumas normas são mais restritivas em relação às outras, esperado, possível devido ao uso dos coeficientes de segurança, mantiveram os valores de cálculo muito abaixo dos valores de ruptura. Este trabalho pretende contribuir para a melhor utilização da combinação das principais normas utilizadas. strengthened with fibre-reinforced polymers (FRP) is a very complex subject. The actual most important codes/recommendations quantify the nominal shear strength a simple sum of the contributions to concrete $\left(V_{c}\right)$, steel $\left(V_{s w}\right)$, and FRP $\left(V_{f}\right)$. This available in literature $N B R$ (1) EUROCODE 2 (1992), ACl 318M (2011), ACI 440-2R (2008), fib BULLETIN 14 - TG 9.3 (2001) and CNR-DT 200 (2004) and analyses the safety factors. Experimental results of 30 RC beams strengthened with PRFC made the database. Some guides are more restrictive in respect of the others, giving a minor contribution to the strengthening therefore, gives more safety. Graphics Comparisons between the experimental and corresponding analical formulations demonstrated the expected below to the collapses values. This work aims to contribute to the best use of the combination of the main codes used.
\end{abstract}




\section{INTRODUÇÃO}

A importância do reforço ao cisalhamento deve-se ao modo de colapso, ruptura frágil, e é considerado crítico uma vez que os colapsos por esforço cortante ocorrem sem advertir de antemão. São mais catastróficos quando comparados com os colapsos por flexão, as quais geralmente são mais progressivos e proporcionam um maior período de tempo antes da ruptura. Estudos já efetuados por Taerwe et al. (1997), Chaallal (1997), Triantafillou (1998), Khalifa et al. (2000) dentre outros, demonstraram que a colagem externa de compósitos de PRFC (Polímeros Reforçados com Fibras de Carbono) na forma de mantas e laminados permitem incrementar a resistência ao esforço cortante em vigas de concreto armado mediante o envolvimento total ou parcial dos elementos. Pellegrino e Modena (2008) analisaram experimentalmente diferentes tipos de colapsos e desenvolveram um novo modelo para a contribuição do PRFC em vigas reforçadas ao cisalhamento.

Aumentando-se a resistência ao cisalhamento pode-se ter como resultado colapsos por flexão, os quais são de natureza mais dúctil quando comparados com os colapsos por cisalhamento. No entanto, as ruínas prematuras, principalmente aquelas causadas pelo descolamento antecipado do PRFC, faz com que o nível máximo de tensão mobilizado no reforço seja bastante inferior que a sua resistência última. Esta preocupação está mobilizando a comunidade científica a desenvolver novas formas de reforços ao esforço cortante utilizando o PRFC.

As pesquisas que foram realizadas para estudar a contribuição do PRFC no aumento da capacidade resistente ao cisalhamento do concreto armado em elementos estruturais foram estudos teóricos com base na adição das parcelas conforme descreve a Equação 1.

$$
V n=V c+V s w+V f
$$

Em que:

$\mathbf{V}_{\mathbf{n}}=$ Resistência do elemento ao cisalhamento $(\mathrm{kN})$; $\mathbf{V}_{\mathbf{c}}=$ Contribuição do concreto ao cisalhamento $(\mathrm{kN})$;
$\mathbf{V}_{\text {sw }}=$ Contribuição da armadura transversal ao cisalhamento $(\mathrm{kN})$;

$\mathbf{V}_{\mathbf{f}}=$ Contribuição do sistema de compósito ao cisalhamento $(\mathrm{kN})$;

$$
\text { As mais tradicionais normas }
$$

internacionais validam a adição da contribuição do PRFC à fórmula anteriormente descrita para o concreto e estribos de aço sugerindo uma segurança adicional. Nos últimos anos, pesquisadores têm empreendido esforços notáveis visando compreender o comportamento do polímero reforçado por fibras (PRF) que foram discutidos por vários autores e basearam as recomendações das mais difundidas normas como ACl 440.2R (2008), fib Bulletin 14-TG 9.3 (2001) e o CNR-DT 200 (2004).

\section{OBJETIVOS}

O presente trabalho visa comparar resultados de dados experimentais com formulações analíticas propostas nas normas e códigos mais difundidos. Desenvolveu-se uma série de comparações entre as normas e recomendações NBR 6118 (2014), EUROCODE 2 (1992), ACl 318M (2011), para o concreto armado, e o ACl 440.2R (2008), fib BULLETIN 14 - TG 9.3 (2001) e CNR-DT 200 (2004) para o PRFC. Através de tabelas, gráficos e análise estatística analisa-se o comportamento de vigas de concreto armado reforçadas ao cisalhamento com PRFC, levando-se em conta os coeficientes de segurança e a contribuição à margem de segurança por eles oferecida para cada combinação.

\section{MODELOS ANALÍTICOS}

Para as várias técnicas de reforço que são utilizadas para aumentar a resistência das vigas ao cisalhamento três configurações principais de colagem se destacam: a colagem do PRFC nas laterais, em três faces em forma de $U$, e o envolvimento total, conforme ilustra a Figura 1. 


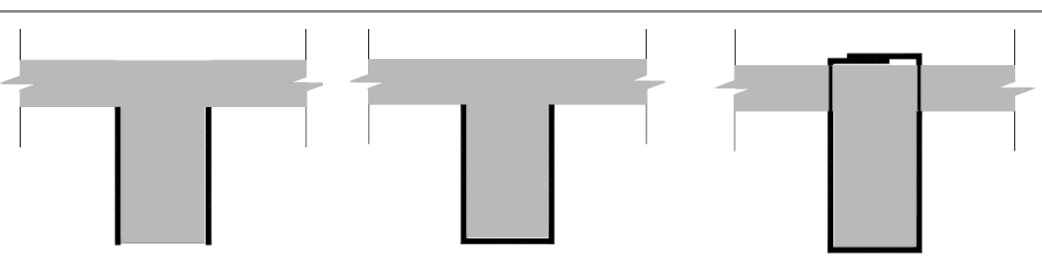

FIGURA 1: Configurações de colagem.

FONTE: $\mathrm{ACl} 440.2 \mathrm{R}$ (2008).

\subsection{ACl 440.2R (2008)}

A resistência nominal ao cisalhamento da viga de concreto armado reforçada com CFRP é determinada pela soma da contribuição do reforço de CFRP com as contribuições do aço e do concreto da seguinte forma, Equação 2:

$$
\varnothing \mathrm{V}_{\mathrm{n}}=\varnothing\left(\mathrm{V}_{\mathrm{c}}+\mathrm{V}_{\mathrm{s}}+\psi_{\mathrm{f}} \mathrm{V}_{\mathrm{f}}\right)
$$

Os fatores de redução recomendados são: $\emptyset=0,85$ e $\psi_{f}=0,95$ para envolvimento total e $\psi_{f}=0,85$ para colagem nas laterais ou em três faces em firma de $U$.

A formulação proposta pela $\mathrm{ACl} 440.2 \mathrm{R}$ (2008), a fim de obter a contribuição do reforço de PRFC à resistência ao cisalhamento total de vigas de concreto, é baseada no trabalho realizado por Khalifa et al. ( 2000). Considera-se uma teoria para o projeto, onde a fratura do PRFC é bastante semelhante à teoria utilizada para calcular a contribuição da armadura de cisalhamento com aço . No entanto, com base na pesquisa realizada por Triantafillou (1998), considerou-se que a ruptura do PRFC acontece em valores mais baixos do que a sua força máxima, devido às concentração de tensões. Khalifa et al. (2000) propõem que a contribuição de cisalhamento do PRFC seja dada pela Equação 3:

$$
V_{f}=\frac{A_{f v} f_{f e}(\sin \alpha+\cos \alpha) d_{f}}{s_{f}}
$$

\section{Em que:}

$\boldsymbol{\alpha}=$ ângulo de inclinação do reforço com PRF;

$\mathbf{S}_{\mathbf{f}}=$ espaçamento entre tiras de PRF;

$\mathbf{d}_{\mathrm{f}}=$ altura útil do PRF;

$A_{f v}=$ área total dos PRF

Para calcular a tensão última de tração no reforço ao cisalhamento de $\operatorname{PRFC}\left(f_{f e}\right) \quad$ que corresponde à única incógnita desconhecida na Equação anterior, o nível de tensão que é a deformação última desenvolvida no reforço de PRFC é calculada pela Equação 4:

$$
f_{\mathrm{fe}}=\varepsilon_{\mathrm{fe}} E_{\mathrm{f}}
$$

\section{Em que:}

$\mathbf{E}_{\mathrm{f}}$ é o módulo de elasticidade e;

$\boldsymbol{\varepsilon}_{\mathrm{fe}}$ é a deformação efetiva, dada por meio das Equações 5 e 6 .

Para barras totalmente envolvidas:

$$
\varepsilon_{\mathrm{fe}}=0,004 \leq 0,75 \varepsilon_{\mathrm{fu}} \quad \text { Eq. [5] }
$$

Para barras com envolvimento em forma de $U$ e colagem nas laterais:

$$
\varepsilon_{\mathrm{fe}}=\mathrm{k}_{\mathrm{v}} \varepsilon_{\mathrm{fu}} \leq 0,004
$$

\section{2 fib BULLETIN 14 - TG 9.3 (2001)}

A capacidade resistente ao cisalhamento de uma viga reforçada é calculada de acordo com o EUROCODE 2 (1992) como mostra a Equação 7:

$$
\begin{array}{r}
\mathrm{V}_{\mathrm{Rd}}=\min \left(\mathrm{V}_{\mathrm{cd}}+\mathrm{V}_{\mathrm{wd}}+\mathrm{V}_{\mathrm{fd}}, \mathrm{V}_{\mathrm{Rd}, 2}\right) \quad \text { Eq. [7] } \\
\mathrm{O} \text { modelo analítico fornecido pela }
\end{array}
$$
fib BULLETIN 14 -TG 9.3 (2001) define a tensão efetiva $\varepsilon_{f e}$, correspondente à tensão do PRF antes do colapso por cisalhamento. Esta tensão multiplicada pelo módulo de elasticidade do PRF na direção da fibra $E_{f}$ e pela área da seção transversal fornece a capacidade total do elemento reforçado como mostra a Equação 8:

$$
\begin{array}{r}
V_{\mathrm{fd}}=0,9 \varepsilon_{\mathrm{fd}, \mathrm{e}} \mathrm{E}_{\mathrm{fu}} \rho_{\mathrm{f}} \mathrm{b}_{\mathrm{w}} \mathrm{d}(\cot \theta+\cot \alpha) \sin \alpha \text { Eq. [8] } \\
\text { Para envolvimento completo ou }
\end{array}
$$
ancoragem apropriada de CFRP o controle de ruptura do FRP é dado pela Equação 9:

$$
\varepsilon_{\mathrm{fe}}=0,17 \cdot\left(\frac{\mathrm{f}_{\mathrm{cm}}^{2 / 3}}{\mathrm{E}_{\mathrm{fu}} \cdot \rho_{\mathrm{f}}}\right)^{0,30} \cdot \varepsilon_{\mathrm{fu}} \quad \text { Eq. [9] }
$$

Para colagem do sistema nas laterais ou em forma de $U$ com CFRP é fornecido pela Equação 10: 


$$
\varepsilon_{\mathrm{fe}}=\min \left[0,65 \cdot\left(\frac{\mathrm{f}_{\mathrm{cm}}^{2 / 3}}{\mathrm{E}_{\mathrm{fu}} \cdot \rho_{\mathrm{f}}}\right)^{0,56} \cdot 10^{-3} ; 0,17\left(\frac{\mathrm{f}_{\mathrm{cm}}^{2 / 3}}{\mathrm{E}_{\mathrm{fu}} \cdot \rho_{\mathrm{f}}}\right)^{0,30} \cdot \varepsilon_{\mathrm{fu}}\right]
$$

As unidades de $\mathrm{f}_{\mathrm{cm}}$ devem ser em MPa e $E_{\text {fu }}$ em GPa.

\section{Em que:}

$\boldsymbol{\rho}_{\mathrm{f}}=$ taxa de reforço;

$\mathbf{t}_{\mathrm{f}}=$ espessura do compósito;

$\mathbf{S}_{\mathbf{f}}=$ espaçamento entre as faixas de reforço ao cisalhamento;

$\mathbf{W}_{\mathbf{f}}=$ largura da faixa de CFRP;

$\mathbf{E}_{\mathrm{f}}=$ módulo de elasticidade do compósito;

$\boldsymbol{\varepsilon}_{\mathrm{fe}}=$ deformação específica do compósito;

$\boldsymbol{\beta}=$ ângulo de inclinação entre a orientação das

fibras e o eixo longitudinal das peças.

Para colapso por aderência (configuração em $U$ ou sem ancoragem) o coeficiente $\gamma_{f}$ é igual a 1,3. Como o valor de $\varepsilon_{\mathrm{fe}}$ é um valor médio, multiplica-se por um valor $\mathrm{k}=0.8$ para se obter o valor característico.

\subsection{CNR-DT 200 (2004)}

Para as recomendações italianas o reforço ao cisalhamento com PRFC deve computar o esforço cortante de acordo com a Equação 11:

$V_{R d}=\min \left(V_{R d, c t}+V_{R d, s}+V_{R d, f}, V_{R d, m a x}\right)$ Eq.[11]

Para a configuração do reforço colado nas laterais utiliza-se a Equação 12, sendo que $\gamma_{R d}$ deve ser igual a 1,2 ou conforme recomenda a norma:

$$
\mathrm{V}_{\mathrm{Rd}, \mathrm{f}}=\frac{1}{\gamma_{\mathrm{Rd}}} \min \left\{0,9 \mathrm{~d}, \mathrm{~h}_{\mathrm{w}}\right\} \mathrm{f}_{\mathrm{fed}} 2 \mathrm{t}_{\mathrm{f}} \frac{\sin \beta \mathrm{w}_{\mathrm{f}}}{\sin \theta \mathrm{s}_{\mathrm{f}}} \quad \text { Eq. [12] }
$$

Para as configurações em U (três lados) e envolvimento total da viga utiliza-se a Equação 13:

$$
V_{R d, f}=\frac{1}{\gamma_{R d}} 0,9 d \cdot f_{f e d} 2 t_{f}(\cot \theta+\cot \beta) \frac{w_{f}}{s_{f}} \quad \text { Eq. [13] }
$$

O ângulo $\theta$ representa a inclinação da fissura diagonal de cisalhamento em relação ao eixo da viga.

\section{METODOLOGIA}

\subsection{MODELOS TEÓRICOS}

Para a avaliação dos modelos teóricos utilizou-se os resultados da pesquisa experimental realizada por Menon (2008), onde foram analisadas experimentalmente 23 vigas com seção transversais retangulares. Todas as vigas foram igualmente dimensionadas: distância entre vãos de $222 \mathrm{~cm}$ e seção transversal de $12 \times 25 \mathrm{~cm}$. As armaduras longitudinais de tração e compressão eram compostas por duas barras de aço CA-50 com $16 \mathrm{~mm}$ de diâmetro. A armadura transversal foi subdimensionada, composta por estribos de aço CA- 60 de 4,2 mm de diâmetro com espaçamento de $18 \mathrm{~cm}$. Dez vigas apresentavam reforços laminados nas laterais, seis vigas laminados em forma de $\mathrm{U}$ e seis vigas foram envolvidas completamente com faixas de tecido e uma foi utilizada como viga referência, sem reforço. No Quadro 1 apresenta-se as características dos materiais constituintes dos sistemas de reforços em PRFC obtidas nas fichas técnicas disponibilizadas pelo fornecedor.

Os cálculos foram realizados utilizando as planilhas do Microsoft Excel, onde, a partir dos cálculos de resistência ao cisalhamento e contribuição do PRFC, realizou-se as diferentes combinações e em seguida confeccionou-se os gráficos. A carga experimental de ruptura das vigas foi comparada com o valor analítico de cálculo obtido através das diversas combinações que forneceu a margem de segurança fornecida pelos coeficientes de segurança.

Para a determinação da contribuição do concreto e da armadura transversal, foram utilizados os critérios da NBR 6118 (2014) modelo I e modelo II; do EUROCODE 2 (1992) e da ACI 318 (2005) , enquanto a contribuição do PRFC foi obtida utilizando-se expressões propostas pelo 


\begin{tabular}{|c|c|c|c|c|}
\hline \multicolumn{5}{|c|}{ QUADRO 1: Características dos sistemas de reforço. } \\
\hline Materiais & $\begin{array}{c}\text { Resistência à tração } \\
\text { (Mpa) }\end{array}$ & $\begin{array}{c}\text { Módulo de } \\
\text { elasticidade (Gpa) }\end{array}$ & $\begin{array}{c}\text { Deformação unit. } \\
\text { Ruptura (\%) }\end{array}$ & Espessura (mm) \\
\hline Laminado & 2800 & 165 & 1,9 & 1,2 \\
\hline Laminado em L & 2250 & 120 & 1,7 & 1,4 \\
\hline Tecido unidirecional & 3450 & 234 & 1,5 & 0,12 \\
\hline
\end{tabular}

FONTE: adaptado de Menon (2008).

QUADRO 2: Combinações das normas referentes de Concreto Armado com as normas de Reforços com PRFC.

\begin{tabular}{|c|c|c|c|}
\hline$V_{c}+V_{s w}$ & $V_{f}$ & $\mathrm{~V}_{\mathrm{c}}+\mathrm{V}_{\mathrm{sw}}$ & $V_{f}$ \\
\hline \multirow{3}{*}{ EUROCODE 2} & Fib 14 & \multirow{3}{*}{ NBR 6118 (Modelo I) } & Fib 14 \\
\hline & $\mathrm{ACl} 440.2 \mathrm{R}$ & & $\mathrm{ACl} 440.2 \mathrm{R}$ \\
\hline & CNR DT 200 & & CNR DT 200 \\
\hline \multirow{3}{*}{$\mathrm{ACl} 380$} & Fib 14 & \multirow{3}{*}{ NBR 6118 (Modelo II) } & Fib 14 \\
\hline & $\mathrm{ACl} 440.2 \mathrm{R}$ & & $\mathrm{ACl} 440.2 \mathrm{R}$ \\
\hline & CNR DT 200 & & CNR DT 200 \\
\hline
\end{tabular}

$\mathrm{ACl} 440.2 \mathrm{R}$ (2008), pelo fib BULLETIN 14 - TG 9.3 Fib (2001) e pelo CNR-DT 200 (2004). Os resultados das combinações das diferentes normas relativas a resistência das vigas ao cisalhamento e do reforço oferecido pelo PRFC foram expostos em tabelas gráficos e em uma análise estatística para melhor visualização das margens de segurança. O coeficiente de variação (CV), calculado como a razão entre o desvio padrão e o valor médio da relação existente entre $\mathrm{o}$ valor teórico $\mathrm{e}$ experimental (Média), pode ser considerado como o parâmetro importante para a avaliação, pois permite a comparação de dados com diferentes valores médios. $\mathrm{O}$ Quadro 2 mostra as combinações das contribuições através da fórmula da adição $V n=V c+V s w+V f$ entre as diferentes normas estudadas.

\section{ANÁLISE DOS RESULTADOS}

Visando avaliar a segurança no equacionamento da contribuição do reforço oferecido pelo PRFC, utilizou-se os dados experimentais obtidos por Menon (2008) executando-se combinações das normas referentes ao equacionamento analítico da contribuição oferecida pelos PRFC $\left(\mathrm{V}_{\mathrm{f}}\right)$ de acordo com as normas fib BULLETIN 14 - TG 9.3 (2001), ACl 440.2R (2008) e CNR-DT 200 (2004) utilizando os coeficientes de segurança propostos pelas mesmas com normas que recomendam resistência ao cisalhamento de vigas para o concreto e aço, EUROCODE 2 (1992), ACl 318 (2005) e NBR 6118 (ABNT, 2014).

\subsection{PRFC LAMINADO COLADO NAS FACES LATERAIS}

Para as vigas com reforço colado nas laterais, os ensaios experimentais mostraram um comportamento irregular por apresentar somente uma opção de ancoragem, de forma que o modo de ruptura fica condicionado ao local onde a fissura crítica cruza a lâmina. Os resultados das combinações entre as diferentes normas relativas à resistência das vigas ao cisalhamento estão expostos em tabelas e gráficos que mostram os valores analíticos e os valores de ruptura experimental. Os valores dos resultados apresentados pelas combinações entre as normas estudadas estão mostrados na Tabela 1.

\subsubsection{Combinação do EUROCODE 2 com fib BULLETIN 14- TG 9.3, CNR-DT 200 e ACl 440.2R}

Para esta combinação foram utilizados os valores de cálculo da combinação entre as normas EUROCODE 2 (2004) para determinação de $V_{c}+V_{s w}$, e as normas fib BULLETIN 14 - TG 9.3 - (2001), CNR-DT 200 (2004) e ACl 440.2R (2008). A margem de segurança fornecida pelos valores de cálculo das normas estudadas é mostrada na Figura 2. 


\begin{tabular}{|c|c|c|c|c|c|c|c|}
\hline \multirow{3}{*}{ VIGA } & \multirow{3}{*}{$\begin{array}{c}\text { CARGA DE } \\
\text { RUPTURA } \\
\text { (kN) }\end{array}$} & \multicolumn{6}{|c|}{$V_{c}+V_{s}+V_{f}(k N)$} \\
\hline & & \multicolumn{3}{|c|}{ EUROCODE } & \multicolumn{3}{|c|}{ ACl 318} \\
\hline & & 14 FIB & $\mathrm{ACl} 440$ & $\begin{array}{c}\text { CNR DT } \\
200 \\
\end{array}$ & $14 \mathrm{FIB}$ & $\mathrm{ACl} 440$ & $\begin{array}{c}\text { CNR DT } \\
200 \\
\end{array}$ \\
\hline V16 & 92,32 & 42,29 & 36,40 & 17,64 & 47,05 & 41,16 & 22,41 \\
\hline V18 & 108,92 & \multicolumn{6}{|c|}{--- } \\
\hline V22 & 115,64 & \multicolumn{6}{|c|}{$V_{c}+V_{s}+V_{f}(k N)$} \\
\hline V26 & 96,32 & \multicolumn{3}{|c|}{ NBR 6118 (1) com } & \multicolumn{3}{|c|}{ NBR 6118 (2) com } \\
\hline V30 & 79,00 & \multirow{2}{*}{14 FIB } & \multirow{2}{*}{$\mathrm{ACl} 440$} & \multirow{2}{*}{$\begin{array}{c}\text { CNR DT } \\
200\end{array}$} & \multirow{2}{*}{14 FIB } & \multirow{2}{*}{$\mathrm{ACl} 440$} & \multirow{2}{*}{$\begin{array}{c}\text { CNR DT } \\
200\end{array}$} \\
\hline V35 & 120,02 & & & & & & \\
\hline V17 & 92,78 & \multirow{4}{*}{46,70} & \multirow{4}{*}{40,81} & \multirow{4}{*}{22,06} & \multirow{4}{*}{46,57} & \multirow{4}{*}{40,68} & \multirow{4}{*}{21,93} \\
\hline V23 & 96,48 & & & & & & \\
\hline V24 & 77,30 & & & & & & \\
\hline V25 & 111,98 & & & & & & \\
\hline
\end{tabular}

FONTE: Autores (2016).

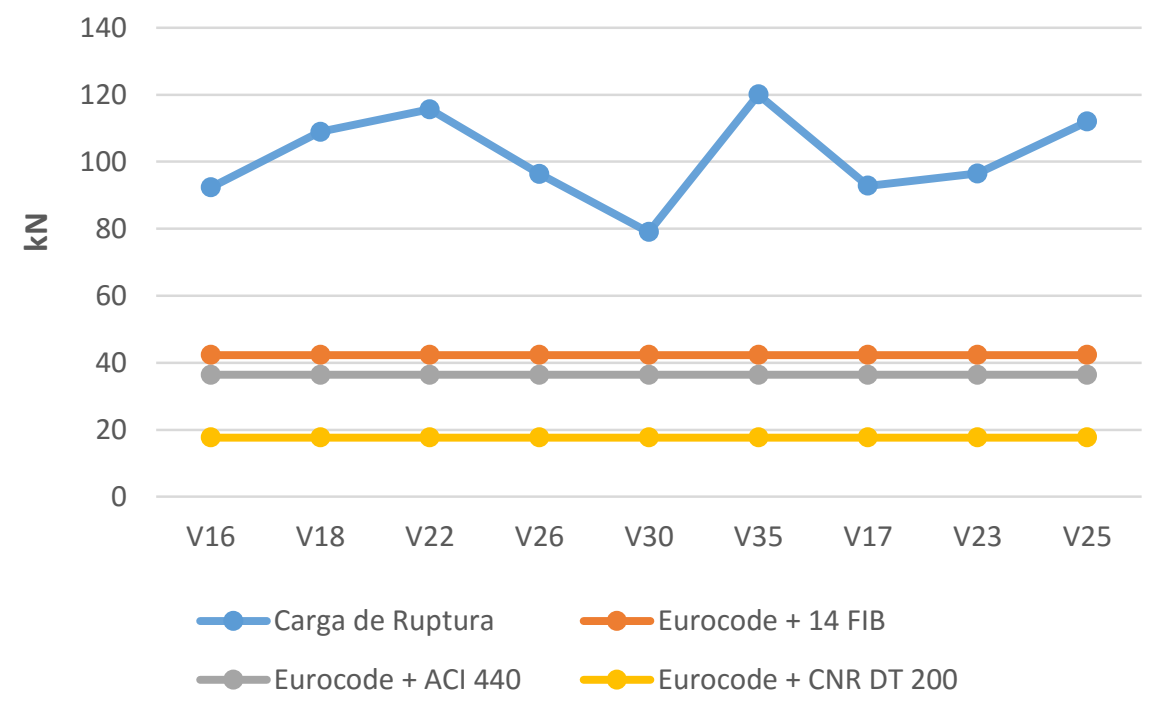

FIGURA 2: Combinações EUROCODE + 14 FIB, EUROCODE + ACl 440 e EUROCODE + CNR DT 200 FONTE: Autores (2016).

O arranjo mais conservador foi 0 calculado pelas normas europeias EUROCODE 2 (1992) e CNR-DT 200 (2004), onde a margem de segurança foi, em todos os casos, maior do que 4,4 . Nota-se também que a combinação de cálculo que mais arrojado aproximando-se do valor da carga de ruptura foi entre as normas EUROCODE 2 (1992) e fib BULLETIN 14 - TG 9.3 - (2001), a qual obteve um valor de $42,29 \mathrm{kN}$ de resistência ao cisalhamento do elemento reforçado. Mesmo assim, pôde-se obter uma margem de segurança de $35,01 \mathrm{kN}$ da viga com carga de ruptura mais baixa, o equivalente a aproximadamente $83 \%$.

\subsubsection{Combinação do ACl 318 com fib BULLETIN 14 - TG 9.3, CNR-DT 200 e ACl 440.2R}

Outro comparativo realizado foi entre os valores de cálculo da combinação entre as normas $\mathrm{ACl} 318$ (2011), para determinação de $\mathrm{V}_{\mathrm{c}}+\mathrm{V}_{\mathrm{sw}}$, e as normas fib BULLETIN 14 - TG 9.3 (2001), CNR-DT 200 (2004) e ACl 440.2R (2008). Conforme o objetivo da pesquisa, a Figura 3 mostra a tolerância entre os valores teóricos e experimentais. 


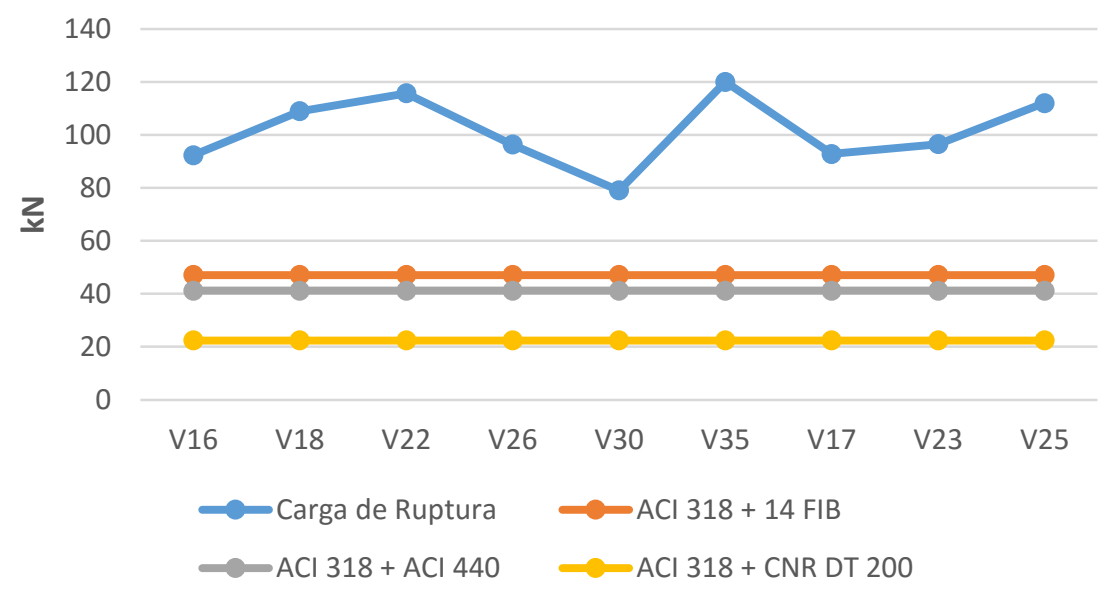

FIGURA 3: Combinações ACl $318+14$ FIB, ACl $318+$ ACl 440 e ACl 318 + CNR DT 200 FONTE: Autores (2016).

Esta combinação apresenta-se mais conservadora e foi a baseada nas normas $\mathrm{ACl} 318$ (2005) e CNR-DT 200 (2004), com resistência ao cisalhamento de $22,41 \mathrm{kN}$, gerando uma margem de segurança de $54,89 \mathrm{kN}$ para a viga que se rompeu com menor carga. Já a combinação que mais se aproximou do valor obtido nos ensaios experimentais foi entre a $\mathrm{ACl} 318$ (2005) e fib BULLETIN 14 - TG 9.3 (2001), o qual apresentou uma margem de segurança de $51,52 \%$ para a viga $\mathrm{V} 24$.

\subsubsection{Combinação da NBR 6118 (modelo I) com fib BULLETIN 14 - TG 9.3, CNR-DT 200 e ACl 440.2R}

Na Figura 4 pode-se observar as variações entre os valores obtidos nos ensaios experimentais e os teóricos com a combinação da norma brasileira NBR 6118 (2014) pelo modelo I, e as normas Fib BULLETIN 14 - TG 9.3 - (2001), CNR-DT 200 (2004) e $\mathrm{ACl} 440.2 \mathrm{R}$ (2008).

A combinação que contém a contribuição de resistência ao cisalhamento calculada pela norma CNR-DT 200 (2004) é a mais conservadora, chegando a uma margem de segurança de $350 \%$ para a viga menos resistente. A combinação composta pela NBR 6118 (2014) no modelo I e a norma Fib BULLETIN 14 - TG 9.3 (2001), foi a que mais se aproximou dos valores apresentados no estudo experimental, portanto a mais arrojada.

\subsubsection{Combinação da NBR 6118 (modelo II) com fib BULLETIN 14 - TG 9.3, CNR-DT 200 e ACl 440.2R}

O comparativo entre os valores obtidos dos ensaios experimentais e as combinações entre a norma NBR 6118 (2014) pelo modelo II, e as normas fib BULLETIN 14 - TG 9.3 - (2001), CNR-DT 200 (2004) e $\mathrm{ACl} 440.2 \mathrm{R}$ (2008), é mostrado na Figura 5.

Neste caso, confirmando as análises anteriores, a combinação que se mostrou mais próxima dos valores estabelecidos pelos ensaios de ruptura foi entre a NBR 6118 (2014) no modelo II e a norma fib BULLETIN 14 - TG 9.3 - (2001), bem como a combinação mais conservadora foi entre a norma brasileira e a CNR-DT 200 (2004).

\subsection{PRFC LAMINADO EM “L” ENVOLVENDO TRÊS FACES DA VIGA (U)}

O segundo estudo foi feito com o sistema de reforço laminado em " $\mathrm{L}$ " posicionado na forma de $U$ em três faces das vigas. Nestes ensaios os resultados foram mais uniformes, o que pode significar que o processo de desprendimento das lâminas iniciou no banzo inferior, fissurado por flexão e cisalhamento.

Os valores dos resultados apresentados pelas combinações entre as normas estudadas estão mostrados na Tabela 2. 


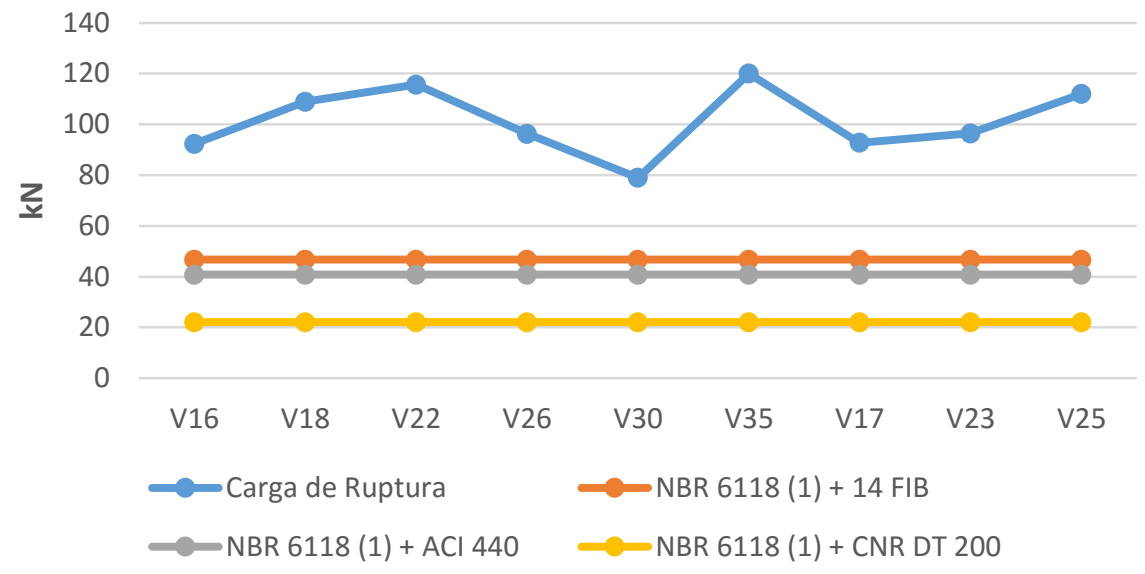

FIGURA 4: Combinações NBR 6118-1 + 14 FIB, NBR 6118-1 + ACI 440 e NBR 6118-1 + CNR DT 200. FONTE: Autores (2016).

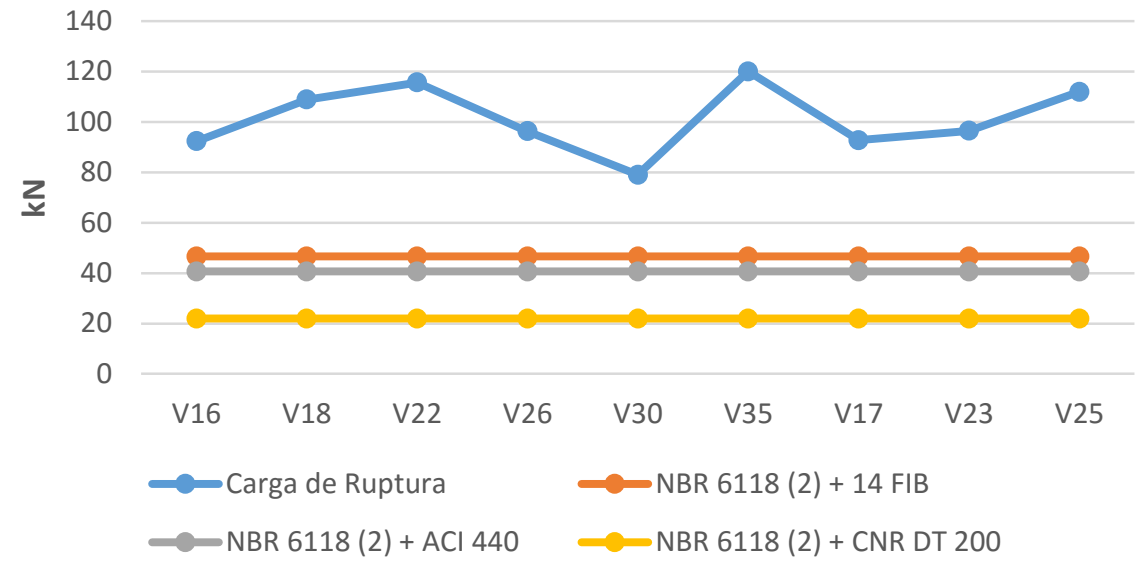

FIGURA 5: Combinações NBR 6118-2 + 14 fib, NBR 6118-2 + ACl 440 e NBR 6118-2 + CNR DT 200. FONTE: Autores (2016).

TABELA 2: PRF laminado em L (Sika CarboShear L) [envolvimento em U].

\begin{tabular}{|c|c|c|c|c|c|c|c|}
\hline \multirow{3}{*}{ VIGA } & \multirow{3}{*}{$\begin{array}{c}\text { CARGA DE } \\
\text { RUPTURA } \\
\text { (kN) }\end{array}$} & \multicolumn{6}{|c|}{$V_{c}+V_{s}+V_{f}(k N)$} \\
\hline & & \multicolumn{3}{|c|}{ EUROCODE } & \multicolumn{3}{|c|}{$\mathrm{ACl} 318$} \\
\hline & & 14 FIB & $\mathrm{ACl} 440$ & CNR DT 200 & $14 \mathrm{FIB}$ & $\mathrm{ACl} 440$ & $\begin{array}{c}\text { CNR DT } \\
200\end{array}$ \\
\hline V19 & 105,22 & 40,33 & 36,84 & 22,33 & 45,10 & 41,60 & 27,09 \\
\hline V20 & 114,43 & --- & --- & --- & --- & --- & --- \\
\hline V21 & 113,19 & \multicolumn{6}{|c|}{$V_{c}+V_{s}+V_{f}(k N)$} \\
\hline V31 & 117,5 & \multicolumn{3}{|c|}{ NBR 6118 (1) } & \multicolumn{3}{|c|}{ NBR 6118 (2) } \\
\hline V32 & 116,6 & 14 FIB & $\mathrm{ACl} 440$ & CNR DT 200 & $14 \mathrm{FIB}$ & $\mathrm{ACl} 440$ & $\begin{array}{c}\text { CNR DT } \\
200\end{array}$ \\
\hline V33 & 115,3 & 44,75 & 41,25 & 26,74 & 44,61 & 41,12 & 26,61 \\
\hline
\end{tabular}




\subsubsection{EUROCODE 2 + fib BULLETIN 14 - TG 9.3, CNR-DT 200 e ACl 440.2R}

Este comparativo foi realizado com base nos valores de cálculo da combinação entre as normas EUROCODE 2 (1992), para determinação de $\mathrm{V}_{\mathrm{c}}+\mathrm{V}_{\mathrm{sw}}$, e as normas fib BULLETIN 14 - TG 9.3 (2001), CNR-DT 200 (2004) e ACl 440.2R (2008). Pode-se observar na Figura 6 que na comparação entre as cargas de ruptura extraídas dos ensaios experimentais e os valores de cálculo das combinações propostas que $o$ arranjo mais conservador foi o calculado pelas normas europeias EUROCODE 2 (1992) e CNR-DT 200 (2004), onde a margem de segurança foi, em todos os casos, maior do que $470 \%$. Nota-se também que a combinação de cálculo que mais se aproximou do valor da carga de ruptura foi entre as normas EUROCODE 2 (1992) fib BULLETIN 14 - TG 9.3 (2001), a qual obteve um valor de 40,33 kN de resistência ao cisalhamento do elemento reforçado. Mesmo assim, pôde-se obter uma margem de segurança de $64,89 \mathrm{kN}$ da viga com carga de ruptura mais baixa, o equivalente a aproximadamente $280 \%$.

\subsubsection{Combinação do ACl 318 com fib BULLETIN} 14 - TG 9.3, CNR-DT 200 e ACl 440.2R

O segundo comparativo do modelo de reforço laminado em " $\mathrm{L}$ " realizado foi entre os valores de cálculo da combinação entre as normas $\mathrm{ACl} 318$ (2011), para determinação de $\mathrm{V}_{\mathrm{c}}+\mathrm{V}_{\mathrm{sw}}$, e as normas fib BULLETIN 14 - TG 9.3 (2001), CNR-DT 200 (2004) e ACl 440.2R (2008). Conforme o objetivo da pesquisa, a Figura 7 mostra a margem de segurança entre os valores teórico e experimental entre as normas estudadas.

Semelhante a comparação anterior, a combinação mais conservadora foi a calculada pelas normas ACl 318 (2005) e CNR-DT 200 (2004), com resistência ao cisalhamento de 27,09 kN, gerando uma margem de segurança de $78,13 \mathrm{kN}$ para a viga que se rompeu com menor carga.

A combinação que mais se aproximou do valor obtido nos ensaios experimentais foi entre a ACI 318 (2005) e fib BULLETIN 14 - TG 9.3 - (2001), o qual apresentou uma margem de segurança de $233 \%$ para a viga V19, sendo esta a que menos resistiu nos ensaios.

\subsubsection{Combinação da NBR 6118 (modelo I) com fib BULLETIN 14 - TG 9.3, CNR-DT 200 e ACl 440.2R}

Este comparativo mostra a combinação da norma brasileira NBR 6118 (2014) modelo I, e as normas fib BULLETIN 14 - TG 9.3 - (2001), CNR-DT 200 (2004) e ACl 440.2R (2008). A Figura 8 mostra as variações entre os valores.

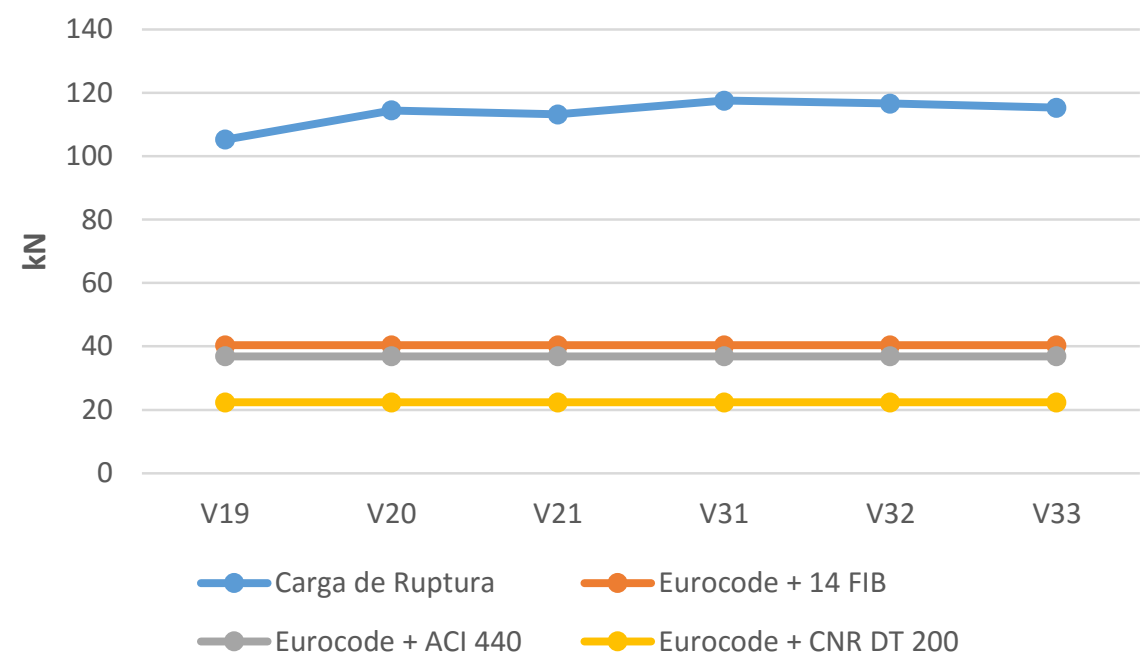

FIGURA 6: Combinações EUROCODE + 14 fib, EUROCODE + ACl 440 e EUROCODE + CNR DT 200. FONTE: Autores (2016). 


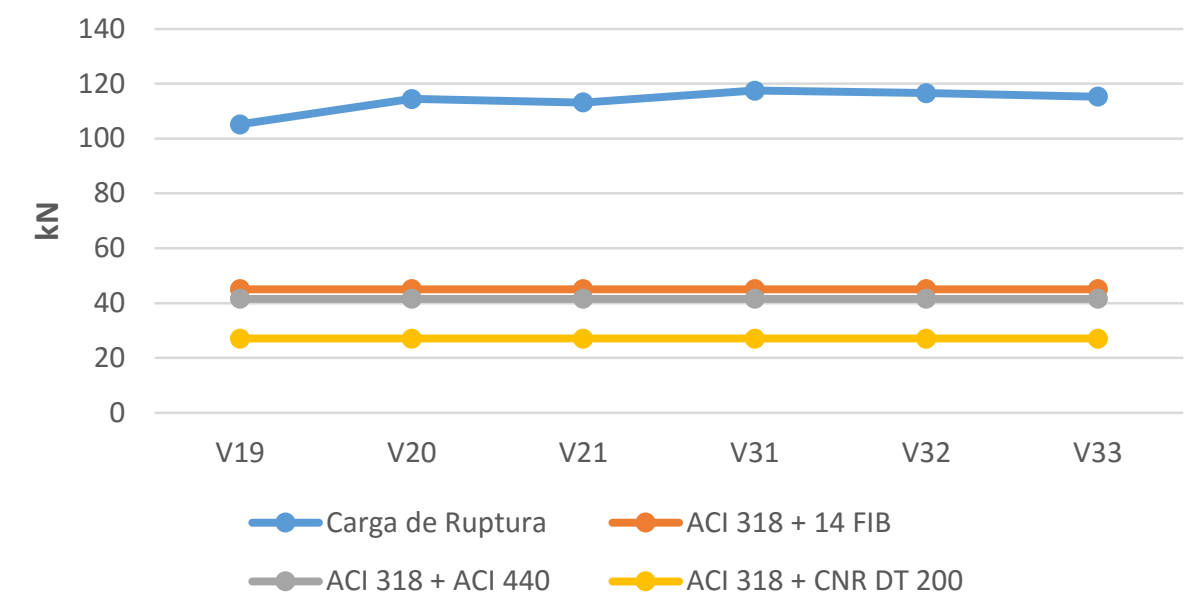

FIGURA 7: Combinações ACl $318+14$ fib, ACl 318 + ACl 440 e ACl 318 + CNR DT 200. FONTE: Autores (2016).

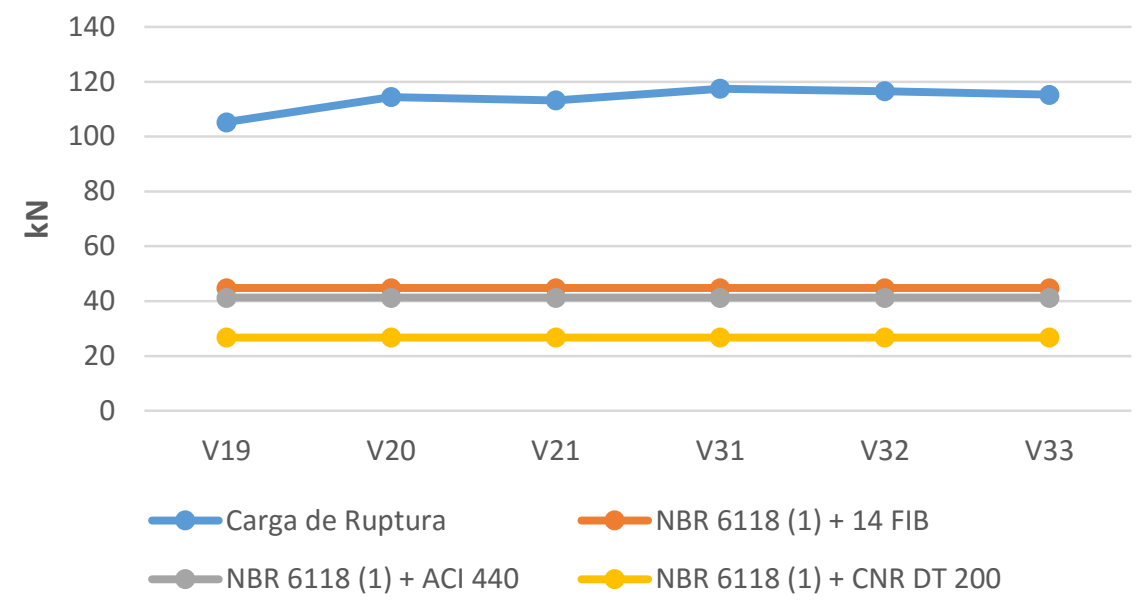

FIGURA 8: Combinações NBR 6118-1 + 14 fib, NBR 6118-1 + ACl 440 e NBR 6118-1 + CNR DT 200. FONTE: Autores (2016).

Verifica-se na Figura 8 que assim como nos casos anteriores, a combinação que contém a contribuição de resistência ao cisalhamento calculada pela norma CNR-DT 200 (2004) é a mais conservadora, chegando a uma margem de segurança de $390 \%$ para a viga menos resistente.

A combinação composta pela NBR 6118 (2014) modelo I e a norma fib BULLETIN 14 - TG 9.3 - (2001), foi a que mais se aproximou dos valores apresentado no estudo experimental.

\subsubsection{Combinação da NBR 6118 (modelo II) com fib BULLETIN 14 - TG 9.3-, CNR-DT 200 e ACI 440.2R}

O último estudo desta série avaliou os valores obtidos dos ensaios experimentais e as combinações entre a norma NBR 6118 (2014) modelo II, e as normas fib BULLETIN 14 - TG 9.3 (2001), CNR-DT 200 (2004) e ACl 440.2R (2008), conforme mostra a Figura 9.

Assim como nas análises anteriores, a combinação que se mostrou mais próxima dos valores estabelecidos pelos ensaios de ruptura foi entre a NBR 6118 (2014) modelo II e a norma fib BULLETIN 14 - TG 9.3 - (2001), bem como a mais conservadora foi entre a norma brasileira e a CNRDT 200 (2004).

\subsection{PRFC EM TECIDO COM ENVOLVIMENTO COMPLETO}

O sistema de reforço que utilizou faixas de tecido de PRFC apresentou desempenho mais uniforme. A pequena variabilidade nos resultados dos protótipos ensaiados pode ser atribuída a uma condição de ancoragem mais eficiente. Os valores dos resultados apresentados pelas combinações entre as normas estudadas estão mostrados na Tabela 3. 


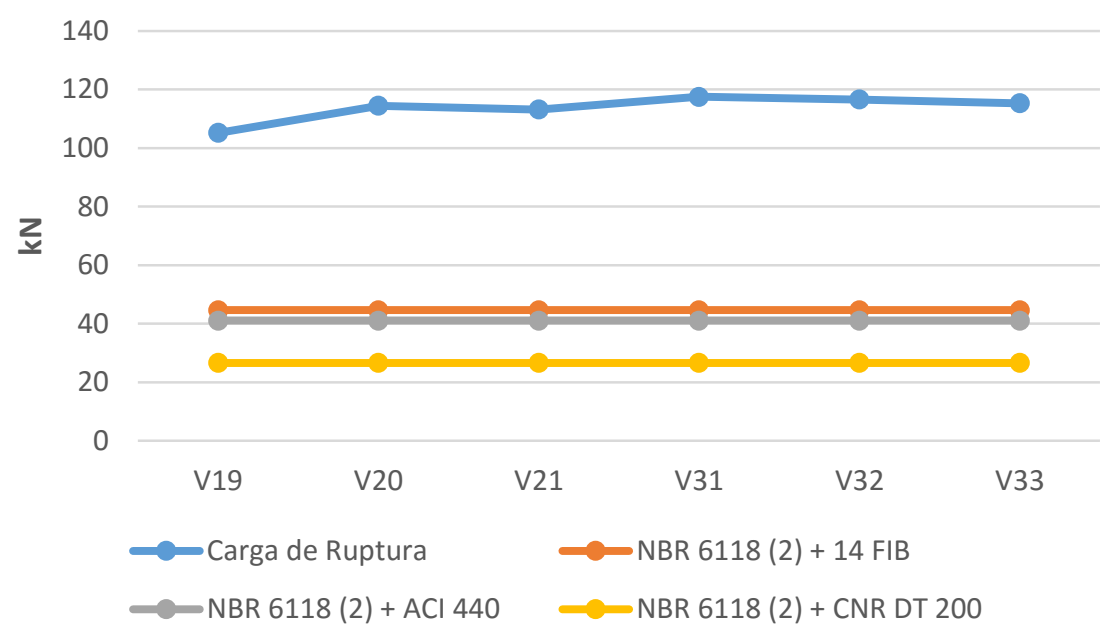

FIGURA 9: Combinações NBR 6118-2 + 14 FIB, NBR 6118-2 + ACI 440 e NBR 6118-2 + CNR DT 200 FONTE: Autores (2016).

TABELA 3: PRFC em tecido (SikaWrap) [envolvimento completo].

\begin{tabular}{|c|c|c|c|c|c|c|c|}
\hline \multirow{3}{*}{ VIGA } & \multirow{3}{*}{$\begin{array}{c}\text { CARGA DE } \\
\text { RUPTURA } \\
\text { (kN) }\end{array}$} & \multicolumn{6}{|c|}{$V_{c}+V_{s}+V_{f}(k N)$} \\
\hline & & \multicolumn{3}{|c|}{ EUROCODE } & \multicolumn{3}{|c|}{$\mathrm{ACl} 318$} \\
\hline & & 14 FIB & $\mathrm{ACl} 440$ & $\begin{array}{c}\text { CNR DT } \\
200\end{array}$ & 14 FIB & $\mathrm{ACl} 440$ & $\begin{array}{c}\text { CNR DT } \\
200\end{array}$ \\
\hline V29 & 120 & 24,93 & 24,33 & 19,05 & 29,70 & 29,10 & 23,81 \\
\hline V37 & 115,6 & --- & --- & --- & --- & --- & --- \\
\hline V28 & 114,4 & \multicolumn{6}{|c|}{$V_{c}+V_{s}+V_{f}(k N)$} \\
\hline V36 & 120,5 & \multicolumn{3}{|c|}{ NBR 6118 (1) } & \multicolumn{3}{|c|}{ NBR 6118 (2) } \\
\hline V34 & 114,6 & $14 \mathrm{FIB}$ & $\mathrm{ACl} 440$ & $\begin{array}{c}\text { CNR DT } \\
200 \\
\end{array}$ & 14 FIB & $\mathrm{ACl} 440$ & $\begin{array}{c}\text { CNR DT } \\
200 \\
\end{array}$ \\
\hline V38 & 118,3 & 29,35 & 28,75 & 23,46 & 29,22 & 28,61 & 23,33 \\
\hline
\end{tabular}

\subsubsection{EUROCODE 2 + fib BULLETIN 14 - TG 9.3-, CNR-DT 200 e ACl 440.2R}

A Figura 10, mostra que na comparação entre as cargas de ruptura extraídas dos ensaios experimentais e os valores de cálculo das combinações propostas. $O$ arranjo mais conservador foi obtido pela combinação das normas europeias EUROCODE 2 (1992) e CNR-DT 200 (2004), onde a margem de segurança foi, em todos os casos, maior do que $600 \%$. Verifica-se também que a combinação de cálculo que mais se aproximou do valor da carga de ruptura foi a combinação das normas EUROCODE 2 (1992) e fib BULLETIN 14 - TG 9.3 (2001), a qual obteve um valor de $24,93 \mathrm{kN}$ de resistência ao cisalhamento do elemento reforçado, entretanto muito abaixo dos valores obtidos nos ensaios experimentais.

\subsubsection{Combinação do ACI 318 com fib BULLETIN 14- TG 9.3, CNR-DT 200 e ACl $440.2 R$}

O segundo comparativo do modelo de reforço em tecido realizado foi entre os valores de cálculo da combinação entre as normas $\mathrm{ACl} 318$ (2011), para determinação de $V_{c}+V_{s w}$, e as normas fib BULLETIN 14 - TG 9.3 - (2001), CNR-DT 200 (2004) e ACl 440.2R (2008). Conforme o objetivo da pesquisa, a Figura 11 mostra a margem de segurança entre os valores teórico e experimental entre as normas estudadas.

Verifica-se, neste caso, que a combinação que mais se aproximou dos valores de ruptura obtidos nos ensaios foi entre as normas $\mathrm{ACl} 318$ (2011) e fib BULLETIN 14 - TG 9.3 - (2001). 


\subsubsection{Combinação da NBR 6118 (modelo I) com} fib BULLETIN 14 - TG 9.3-, CNR-DT 200 e ACl 440.2R

Neste comparativo foi realizado com a combinação da norma brasileira NBR 6118 (2014) modelo I, e as normas fib BULLETIN 14 - TG 9.3(2001), CNR-DT 200 (2004) e ACl 440.2R (2008). A Figura 12 mostra que, assim como as demais combinações, a norma que se apresenta como mais conservadora é a CNR-DT 200 (2004).

\subsubsection{Combinação da NBR 6118 (modelo II) com} fib BULLETIN 14 - TG 9.3, CNR-DT 200 e ACl 440.2R

O comparativo entre os valores obtidos

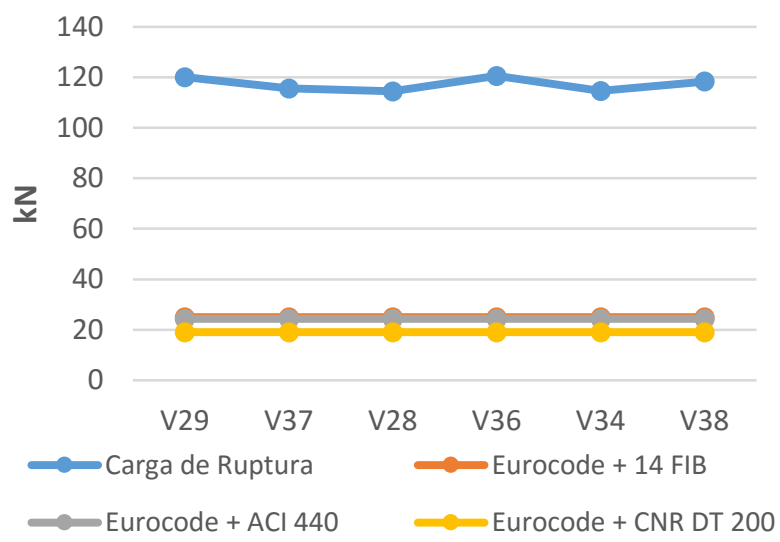

FIGURA 10: Combinações Eurocode + 14 FIB, Eurocode + ACl 440 e Eurocode + CNR DT 200.

FONTE: Autores (2016).

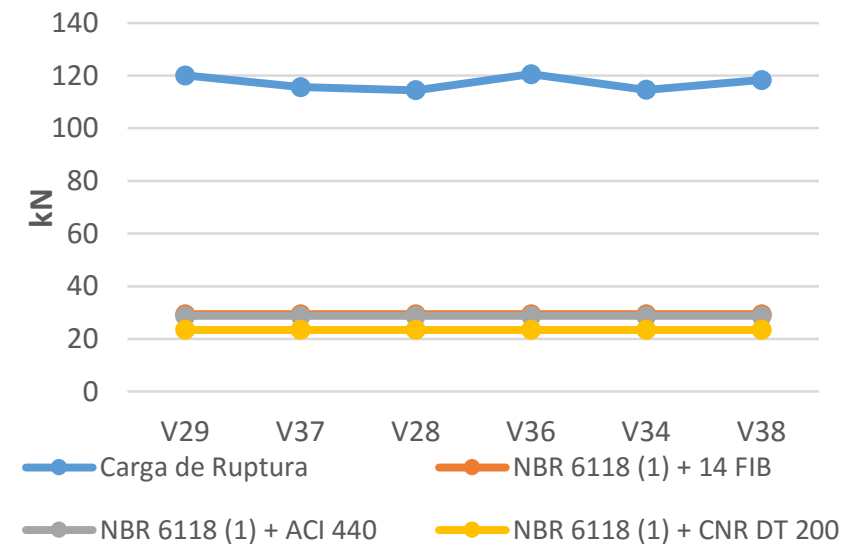

FIGURA 12: Combinações NBR 6118-1 + 14 fib, NBR 6118-1 + ACl 440 e NBR 6118-1 + CNR DT 200. FONTE: Autores (2016). dos ensaios experimentais e as combinações entre a norma NBR 6118 (2014) modelo II, e as normas fib BULLETIN 14 - TG 9.3- (2001), CNR-DT 200 (2004) e ACl 440.2R (2008), são mostrados na Figura 13.

Como nas análises anteriores, a combinação que se mostrou mais próxima dos valores estabelecidos pelos ensaios de ruptura foi entre a NBR 6118 (ABNT, 2014) no modelo II e a norma fib BULLETIN 14 TG 9.3 - (2001), bem como a mais conservadora foi entre a norma brasileira e a CNR-DT 200 (2004).

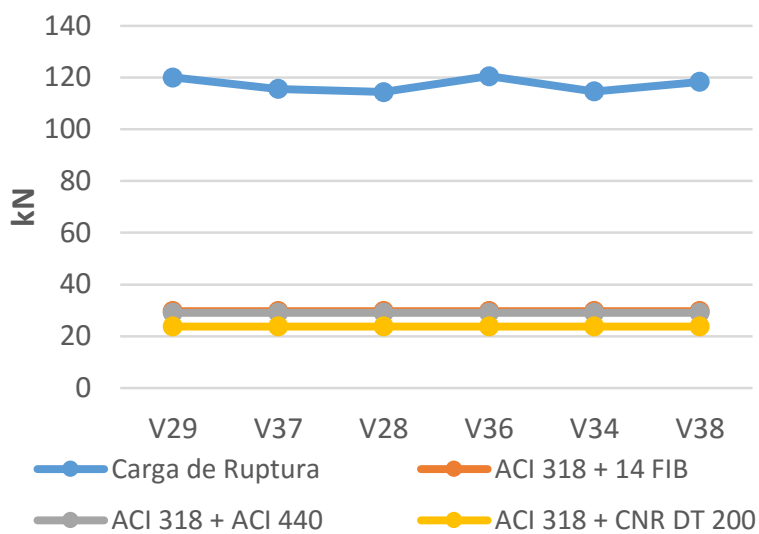

FIGURA 11: Combinações ACI $318+14$ fib, ACI 318 + ACI 440 e ACI 318 + CNR DT 200. FONTE: Autores (2016).

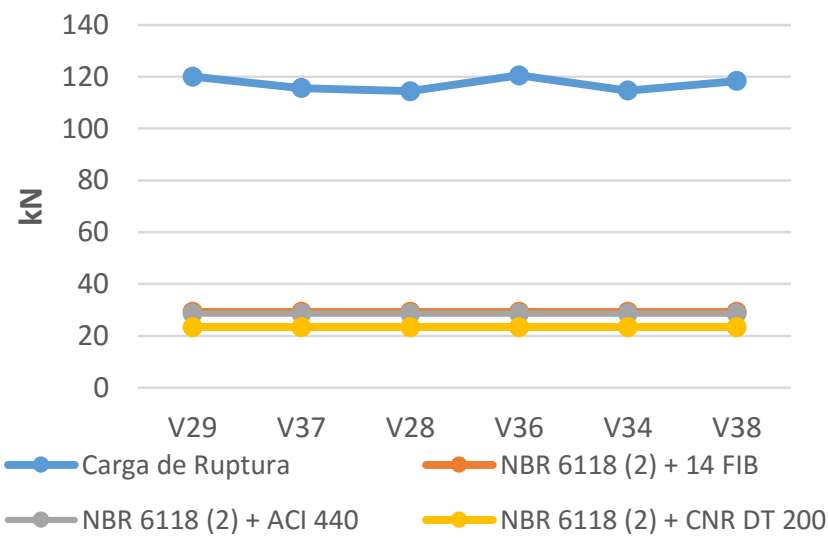

FIGURA 13: Combinações NBR 6118-2 + 14 fib, NBR 6118-2 + ACl 440 e NBR 6118-2 + CNR DT 200. FONTE: Autores (2016). 


\subsection{ANÁLISE ESTATÍSTICA}

Para melhor avaliar e reforçar a conclusão para os principais resultados foi feita uma análise estatística. $\mathrm{O}$ o valor do coeficiente de variação (CV) juntamente com valor médio correspondente a razão entre o valor teórico e experimental (Média) são resumidos na Tabela 4.

\section{CONCLUSÃO}

Conclui-se que os coeficientes de segurança sugeridos pelas diversas normas avaliadas têm grande importância, pois foram efetuados estudos com modelos analíticos sem os coeficientes de segurança e os valores dos esforços cortantes atingiram na sua maioria o valor de colapso. Desta forma os coeficientes de segurança propostos pelas normas conferem ao projetista uma margem de proteção que variam de acordo com o tipo de reforço e com a combinação utilizada em relação aos valores das cargas de ruptura obtidos experimentalmente.

Os gráficos tiveram o comportamento esperado, mantendo sempre os valores de cálculo abaixo dos valores de ruptura, comportamento este, somente possível devido ao uso dos coeficientes de segurança.

O EUROCODE 2 (2004) não foi utilizado pois fornece valores analíticos que atingem os experimentais conforme comprovado na literatura.

Para os laminados colados nas laterais a combinação EUROCODE 2 (1992) e CNR DT 200 (2004) apresentou-se mais conservadora enquanto que a mais arrojada foi a $\mathrm{ACl} 318$ (2005) com a o fib BULLETIN 14-TG 9.3 (2001). As margens de segurança para a configuração de reforço em $U$, apresentaram melhores resultados para a

TABELA 4: Análise Estatística.

PRFCs LAMINADOS (Sika CarboDur S512) [colagem nas laterais]

\begin{tabular}{|c|c|c|c|c|c|c|}
\hline & \multicolumn{2}{|c|}{$14 \mathrm{fib}$} & \multicolumn{2}{|c|}{$\mathrm{ACl} 440$} & \multicolumn{2}{|c|}{ CNR DT 200} \\
\hline & CV & Média & CV & Média & $\mathrm{CV}$ & Média \\
\hline Eurocode & 0,44 & 2,40 & 0,50 & 2,79 & 0,74 & 5,75 \\
\hline $\mathrm{ACl} 318$ & 0,40 & 2,12 & 0,45 & 2,50 & 0,67 & 4,60 \\
\hline NBR 6118 - Modelo I & 0,40 & 2,17 & 0,46 & 2,49 & 0,68 & 4,6 \\
\hline NBR 6118 - Modelo II & 0,40 & 2,18 & 0,46 & 2,50 & 0,68 & 4,63 \\
\hline \multicolumn{7}{|c|}{ PRFCs em tecidos (SikaWrap) [envolvimento completo] } \\
\hline & \multicolumn{2}{|c|}{14 fib } & \multicolumn{2}{|c|}{$\mathrm{ACl} 440$} & \multicolumn{2}{|c|}{ CNR DT 200} \\
\hline & CV & Média & CV & Média & CV & Média \\
\hline Eurocode & 0,50 & 2,82 & 0,54 & 3,10 & 0,70 & 5,09 \\
\hline $\mathrm{ACl} 318$ & 0,45 & 2,52 & 0,49 & 2,73 & 0,64 & 4,20 \\
\hline NBR 6118 - Modelo I & 0,46 & 2,54 & 0,49 & 2,76 & 0,65 & 4,25 \\
\hline NBR 6118 - Modelo II & 0,46 & 2,55 & 0,49 & 2,77 & 0,65 & 4,27 \\
\hline \multicolumn{7}{|c|}{ PRFCs em tecidos (SikaWrap) [envolvimento completo] } \\
\hline & \multicolumn{2}{|c|}{$14 \mathrm{fib}$} & \multicolumn{2}{|c|}{$\mathrm{ACl} 440$} & \multicolumn{2}{|c|}{ CNR DT 200} \\
\hline & CV & Média & CV & Média & $\mathrm{CV}$ & Média \\
\hline Eurocode & 0,68 & 4,7 & 0,69 & 4,82 & 0,75 & 6,15 \\
\hline $\mathrm{ACl} 318$ & 0,62 & 3,95 & 0,63 & 4,03 & 0,69 & 4,92 \\
\hline NBR 6118 - Modelo I & 0,63 & 3,99 & 0,63 & 4,08 & 0,70 & 5,00 \\
\hline NBR 6118 - Modelo II & 0,63 & 4,01 & 0,64 & 4,10 & 0,70 & 5,03 \\
\hline
\end{tabular}


combinação EUROCODE 2 e CNR DT 200, entretanto, as demais não apresentaram grandes desvantagens. Para a configuração composta por faixas de tecido envolvendo totalmente os lados da viga, os resultados foram semelhantes aos dos laminados em forma de U. Ressalta-se que a NBR 6118 (2014) apresenta combinação mais conservativa com a norma italiana CNR DT 200 (2004) e pouco menos conservativa com o fib BULLETIN 14 - TG 9.3 (2001) para ambos os modelos 1 e 2.

O modelo analítico proposto pelo fib BULLETIN 14-TG 9.3 (2001) fornece resultados mais homogêneos em termos de CV para as vigas em todos os casos : colagem na lateral, envolvimento em $U$ e total.

O modelo da norma italiana CNR DT 200 (2004) apresenta resultados relativamente bons em termos de CV para a maioria dos casos de vigas em geral e fornece previsões conservadoras.

O modelo proposto pelo $\mathrm{ACl} 440.2 \mathrm{R}$ (2008) fornece resultados relativamente bons em termos de CV ficando entre o fib BULLETIN 14-TG 9.3 (2001) e o CNR DT 200 (2004).

Como não existe ainda no Brasil, norma para reforços estruturais utilizando-se polímeros reforçados com fibras de carbono, projetistas e pesquisadores utilizam normas internacionais. Espera-se que este trabalho contribua para a melhor utilização da combinação das principais normas utilizadas.

\section{REFERÊNCIAS BIBLIOGRÁFICAS}

AMERICAN CONCRETE INSTITUTE (ACI): Building code requirements for structural concrete $(\mathrm{ACl}$ 318-05) and commentary (318R-05), $\mathrm{ACl}$ 318, Farmington Hills, Mich., USA, 2005.

AMERICAN CONCRETE INSTITUTE (ACI), Guide for the design and construction of externally bonded FRP systems for strengthening of concrete structure, $\mathrm{ACl}$ 440.2R-08, Farmington Hill, Mich., 2008.

ASSOCIAÇÃO BRASILEIRA DE NORMAS TÉCNICAS, NBR6118: Projeto de Estruturas de Concreto. Rio de Janeiro; 2014.
CEN, Eurocode 2: Design of Concrete Structures - Part 11: General Rules and Rules for Buildings, ENV 1992-1-1 1992, Comité Européen d e Normalisation, Brussels. Belgium, 1992.

CEN, Eurocode 2: Design of Concrete Structures - Part 11: General Rules and Rules for Buildings, ENV 1992-1-1 2004, Comité Européen de Normalisation, Brussels. Belgium, 2004.

CHAALLAL, O, Shear Strengthening of RC Beams by Externally Bonded Side CFRP Strips. Journal of Construction, vol. 2, n² 2. May, 1997.pp. 111-113.

CONSIGLIO NAZIONALE DELLE RICERCHE (CNR), Instructions for design, execution and control of strengthening interventions through fiber-reinforced composites. CNR-DT 200-04, Consiglio Nazionale delle Ricerche, Rome, Italy, 2004.

FÉDÈRATION INTERNATIONALE DU BÉTON. Externally Bonded FRP Reinforcement for RC Structures. Technical report by task group 9.3 FRP reinforcement for concrete structures, Fédération Internationale du Béton - fib, Bulletin 14,. Lausanne Switzerland. , 2001.

KHALIFA, A., L. De LORENZIS, and A. NANNI, FRP Composites for Shear Strengtheneing of RC Beams, Proc., 3rd Inter. Conf. on Advanced Composite Materials in Bridges and Structures, Ottawa, Canada, J. Humar and A.G. Razaqpur, Editors, 15-18 Aug. 2000, pp. 137-144.

MENON, N.V. Estudo Experimental de Sistemas de Reforço ao Cisalhamento em Vigas de Concreto Armado Utilizando-se Polímero Reforçado com Fibras de Carbono (PRFC). (Tese) Doutorado em Estruturas, Departamento de Engenharia Civil, Universidade Federal de Santa Catarina, Florianópolis, Brasil, 2008, 295 p.

PELLEGRINO C., MODENA C., An experimentally based analytical model for shear capacity of FRP strengthened reinforced concrete beams, Mechanics of Composite Materials, Vol. 44, n. 3, 2008, pp. 231-244.

TAERWE, L., KHALL, H., MATTHYS, S. Behavior or RC Beams Strengthened in Shear by External CFRP Sheets Non-metallic (FRP) reinforcement for concrete structures. vol 1, Japan Concrete Institute. Tokyo.1997. pp 487-490

TRIANTAFILLOU, T. Shear Strengthening of Reinforced Concrete Beams Using Epoxy-Bonded FRP Composites. ACl Structural Journal, March-April, 1998. pp 107-115. 